470 Troeger u. Grothe: Ueb.d. beiden Thiosulfonsäuren ete.

\title{
Ueber die beiden Thiosulfonsäuren des Naphtalins sowie 0-Toluolthiosnlfonsäure;
}

von

\section{J. Troeger und W. Grothe.}

(Aus dem Laboratorium für synthetische und pharmaceutische Chemie der technischen Hochschule zu Braunschweig.)

Von den Thiosulfonsäuren der Benzolreihe sinð nur die Thiosulfonsäuren des Benzols und des Toluols (1.4) in Form ihrer Salze bekannt. Dieselben entstehen bei der Einwirkung von wässriger Kaliumsulfidlösung auf die Chloride der entsprechenden Sulfonsäuren oder durch längere Behandlung der sulfinsauren Salze mit Schwefel. Die Salze dieser beiden bisher bekannten Thiosulfonsäuren lassen sich durch Umkrystallisiren aus Alkohol sehr leicht reinigen und liefern hierbei ausgezeichnet krystallisirende Produkte. Da wir zu einer experimentellen Untersuchung, über die später ausführlich berichtet werden wird, weitere Repräsentanten von Thiosulfonsäuren benöthigten, so haben wir uns mit der weiteren Darstellung der Thiosulfonsäuren des Naphtalins und Toluols (1.2) befasst. Vorausgeschickt sei, dass die Gewinnung der reinen Salze dieser Säuren vielerlei Schwierigkeiten bietet, da ihnen die ausserordentliche Krystallisationsfähigkeit, die den thiosulfonsauren Salzen des Benzols und Toluols (1.4) eigen ist, vollständig abgeht. Sie sind in den zur Reinigung üblichen Mitteln, wie Wasser oder Alkohol, entweder zu schwer oder zu leicht löslich, so dass eine Trennung von dem bei der Umsetzung gemäss der nachstehenden Gleichung gebildeten Chlorkalium,

$$
\text { (R). } \mathrm{SO}_{2} \mathrm{Cl}+\mathrm{K}_{2} \mathrm{~S}=(\mathrm{R}) \mathrm{SO}_{2} \mathrm{SK}+\mathrm{KCl} \text {, }
$$

auf grosse Schwierigkeiten stösst. Für die meisten $Z$ wecke und speciell auch für unsere mit diesen thiosulfonsauren Salzen geplanten Versuche ist eine geringe Beimengung ron Chlorkalium ohne Bedeutung; handelt es sich aber um die Beschaffung eines analysenreinen Materials, so ist eventuell durch wiederholte fractionirte Krystallisation das thiosulfonsaure Salz von dem beigemengten Chlorkalium zu trennen. Bei der Darstellung der thiosulfonsauren Salze des Naphtalins wurde auch 
der zweite Weg, d. h. die Anlagerung von Schwefel an Sulfnate, versucht, jedoch ohne Erfolg. So lange es sich daher um die Beschaffung eines thiosulfonsauren Salzes, dem etwas Chlorkalium beigemengt sein darf, handelt, treten wesentliche Schwierigkeiten nicht entgegen; dieselben stellen sich erst ein, sobald ein absolut reines Salz verlangt wird.

$a-N$ aphtalinthiosulfonsäure.

Das Salz dieser Säure bildet sich bei der Einwirkung von Kaliumsulfid auf $\alpha$-Naphtalinsulfonchlorid. Es empfiehlt sich, hier alkoholisches Kaliumsulfid anzuwenden und das fein zerriebene Chlorid in kleinen Antheilen in die Sulfidlösung einzutragen. Anfangs thut man gut, nach dem jemaligen Eintragen des Chlorids gelinde zu erwärmen, damit eine vollständige Umsetzung des Chlorids erfolgt. Beim weiteren Eintragen des Chlorids wird die Wärmezufuhr überflüssig, da die bei dem Prozesse auftretende Reactionswärme schliesslich ausreichend ist. Ist der Process im Gange, so beobachtet man beim jedesmaligen Eintragen des Chlorids eine milchige Trübung, die beim Umschütteln sofort wieder verschwindet. Diese Trübung rührt wahrscheinlich von ausgeschiedenem Schwefel ber, der gemäss der nachstehenden Gleichung neben sulfinsaurem Salz sich abscheidet und erst im weiteren Verlauf der Reaction die Bildung des thiosulfonsauren Salzes bedingt:

$\mathrm{C}_{10} \mathrm{H}_{7} \mathrm{SO}_{2} \mathrm{Cl}+\mathrm{K}_{8} \mathrm{~S}=\mathrm{C}_{10} \mathrm{H}_{7} \mathrm{SO}_{2} \mathrm{~K}+\mathrm{S}+\mathrm{KCl}=\mathrm{C}_{10} \mathrm{H}_{7} \mathrm{SO}_{2} \mathrm{SK}+\mathrm{KCl}$.

Das Ende der Reaction, d. h. die für eine beliebige Menge Kaliumsulfidlösung nöthige Menge Chlorid, lässt sich leicht daran erkennen, dass die ziemlich dunkel gefärbte Flüssigkeit sich stark milchig trübt. Bei einiger Uebung lässt sich dieser Punkt ziemlich leicht treffen, und man erhält beim Umschütteln eine dunkle, neutrale Lösung, in der das bei dem Processe gebilde Chlorkalium suspendirt ist. Sollte man aus Versehen etwas zu viel Chlorid eingetragen haben, was an der sauren Reaction der Lösung sich zu erkennen giebt, so kann man durch nachträgliches Zufügen von Kaliumsulfidlösung leicht die neutrale Reaction herstellen. Trennt man nunmehr die alkoholische, neutrale Lösung von dem Chlorkalium durch Filtriren, so beginnt bei genügender Concentration der alkoholischen Lösung die Abscheidung von Krystallblättchen. Die 
so gewonnenen Krystalle sind das Kaliumsalz der $\alpha$-Naphtalinthiosulfonsäure. Sie bilden schmutzig weisse Blättchen, die krystallwasserfrei sind. In Wasser ist das thiosulfonsaure Salz bedeutend schwerer löslich, als das entsprechende sulfinsaure Salz. Verhältnissmässig schwer löslich ist das Salz auch in Alkohol, aus dem es behufs weiterer Reinigung zweckmässig umkrystallisirt wird. Lässt man die alkoholischen Mutterlaugen stehen, so scheiden sich weitere Mengen des Salzes ab, beim Einengen der alkoholischen Mutterlaugen tritt abermals Salzabscheidung ein und beim Erkalten erstarrt dann die Lösung zu einem Krystallbrei. Auf diese Weise erklärt es sich, dass, wenn man bei einer möglichst guten Ausbeute ein reines $\mathrm{Salz}$ gewinnen will, man auf grosse Schwierigkeiten stösst.

Die einzelnen, zuweilen analysirten Krystalle zeigten im Kaliumgehalt Schwankungen von $15,48 \%-17,5 \% \mathrm{~K}$, während die Formel $\mathrm{C}_{10} \mathrm{H}_{7} \mathrm{SO}_{2} \mathrm{SK}$ einen Kaliumgehalt von $14,89 \%$ erfordert.

Dass aber durch Umkrystallisiren aus Alkohol, bezw. durch fractionirte Krystallisation eine Reinigung des Salzes möglich ist, beweist die nachstehende Analyse.

$0,3974 \mathrm{Grm}$. Substanz gaben $0,1310 \mathrm{Grm} . \mathrm{K}_{2} \mathrm{SO}_{4}$, entsprechend $0,058701 \mathrm{Grm} . \mathrm{K}=14,77 \% \mathrm{~K}$. $14,89 \%$.

Für die Formel $\mathrm{C}_{10} \mathrm{H}_{7} \mathrm{SO}_{2} \mathrm{SK}$ ergiebt sich ein Kaliumgehalt von

$$
\beta \text {-Naphtalinthiosulfonsäure. }
$$

Die Darstellung des Kalisalzes dieser Säure geschah in ganz derselben Weise, wie wir es bei der entsprechenden $\alpha$-Säure beschrieben haben. Nachstehend sind einige Analysen angeführt, die von Salzen herrühren, welche nach dem oben erläuterten Verfahren direct, ohne nochmaliges Umkrystallisiren, gewonnen sind. Bei allen Analysen zeigt sich auch hier durchschnittlich ein zu hoher Kaliumgehalt, der seine Erklärung in einer durch Chlorkalium verursachten Verunreinigung findet. Analysen:

1. 1,7824 Grm. Substanz gaben $0,6224 \mathrm{Grm} . \mathrm{K}_{2} \mathrm{SO}_{4}$, entsprechend $0,27885 \mathrm{Grm} . \mathrm{K}=15,6 \% \mathrm{~K}$.

2. $0,3410 \mathrm{Grm}$. Substanz gaben $0,1176 \mathrm{Grm} . \mathrm{K}_{2} \mathrm{SO}_{4}$, entsprechend $0,052717 \mathrm{Grm} . \mathrm{K}=15,45 \% \mathrm{~K}$.

3. 0,2828 Grm. Substanz gaben $0,0953 \mathrm{Grm} . \mathrm{K}_{2} \mathrm{SO}_{4}$, entsprechend 0,04272 Grm. $\mathrm{K}=15,1 \% \mathrm{~K}$. 
4. 0,3222 Grm. Substanz gaben 0,1092 Grm. $\mathrm{K}_{2} \mathrm{SO}_{4}$, entsprechend $0,48968 \mathrm{Grm} . \mathrm{K}=15.2 \% \mathrm{~K}$.

Das unter 4 analysirte Produkt gab nach nochmaligem Umkrystallisiren kleine, weisse Krystallblättchen, die wasserfrei waren und deren Analyse nachfolgende Werthe ergab:

0,9472 Grm. Substanz gaben $0,3064 \mathrm{Grm}$. $\mathrm{K}_{2} \mathrm{SO}_{4}$, entsprechend $0,1373 \mathrm{Grm} . \mathrm{K}=14,5 \% \mathrm{~K}$.

Da eine Schwefelbestimmung ein viel schärferes Kriterium für das Vorhandensein eines thiosulfonsauren Salzes bot, so haben wir mit dem durch Krystallisation gereinigten Salze noch eine solche ausgeführt.

0,1641 Grm. Substanz gaben 0,2934 Grm. $\mathrm{BaSO}_{4}$, entsprechend 0,04029 Grm. $\mathrm{S}=24,55 \% \mathrm{~S}$.

Die Formel $\mathrm{C}_{10} \mathrm{H}_{7} \mathrm{SO}_{2} \mathrm{SK}$ verlangt $14,89 \% \mathrm{~K}$ und $24,43 \% \mathrm{~S}$.

\section{o-Toluolthiosulfonsäure.}

Das Natriumsalz dieser Säure wurde wie folgt dargestellt. o-Toluolsulfonchlorid wurde in eine concentrirte, wässrige Natriumsulfidlösung nach und nach eingetragen. Hierbei war zum Unterschied von dem entsprechenden p-Toluolsulfonchlorid absolut keine Reaction bemerkbar, sondern das Chlorid blieb unverändert in der Flüssigkeit und erst starkes Erwärmen bewirkte eine Umsetzung, indem unter Aufnahme des Chlorides die Flüssigkeit eine ziemlich dunkle Färbung annahm. Nach öfterem Eintragen des Chlorides unter jeweiligem Erwärmen tritt schliesslich ein Punkt ein, wo die Reactionswärme so gross wird, dass jede weitere Wärmezufuhr unnöthig wird. Trägt man jetzt Chlorid ein, so beobachtet man heftige Reaction mit starker Schwefelabscheidung, die aber beim Umschütteln wieder verschwindet. Das Eintragen des Chlorides erfolgt nun so lange, bis eine starke Graufärbung und Trübung eintritt, d. h. bis die Flüssigkeit schwach saure Reaction zeigt. Alsdann neutralisirt man die Flüssigkeit mittelst etwas Schwefelnatriumlösung und dunstet die tief braun gefärbte Lösung bis zur Trockne ein. Durch Ausziehen mit Alkohol, in dem das Natriumsalz der o-Toluolthiosulfonsäure äusserst leicht löslich ist, trennt man jetzt von dem beigemengten Chlorkalium und engt dann die alkoholische Lösung ein, bis das Ganze zu einem Krystallbrei gesteht. Eine Reinigung durch Umkrystallisiren 
474 Troeger u. Grothe: Ueb.d. beiden Thiosulfonsäuren etc.

aus Wasser oder Alkohol schlug völlig fehl, da in beiden Lösungsmitteln das Salz mit ausserordentlicher Leichtigkeit löslich ist. Die einzige Reinigung, die uns noch übrig blieb, war, das Salz von der braunen Mutterlauge zu befreien und dies geschah nach dem Absaugen des Salzes durch wiederholtes Auswaschen mit Aether. Man gewinnt so ein Salz, das nach dem Trocknen ein schmutziggraues Krystallpulver darstellt. Die folgenden Analysen beweisen die Bildung des thiosulfonsauren Salzes.

Analysen des bei $105^{0}$ getrockneten Salzes.

1. 1,0135 Grm. Substanz gaben $0,3563 \mathrm{Grm} . \mathrm{Na}_{2} \mathrm{SO}_{4}$, entsprechend 0,1147 Grm. $\mathrm{Na}=11,3 \% \mathrm{Na}$.

2. 1,4323 Grm. Substanz gaben 0,5018 Grm. $\mathrm{Na}_{2} \mathrm{SO}_{4}$, entsprechend 0,16255 Grm. $\mathrm{Na}=11,3 \% \mathrm{Na}$.

Die Formel $\mathrm{C}_{7} \mathrm{H}_{7} \mathrm{SO}_{2} \mathrm{SNa}$ verlangt $10,95 \% \mathrm{Na}$.

Wenn auch die Analysenresultate einen etwas zu hohen Natriumgehalt ergaben, so ist doch in Anbetracht einer mangelnden Rninigungsmethode kein $Z$ weifel, dass das vorliegende Salz das Natriumsalz der o-Toluolthiosulfonsäure ist.

Etwas günstiger als die Darstellung des Natriumsalzes verläuft diejenige des Kaliumsalzes, wenn man das o-Toluolsulfonchlorid auf eine alkoholische Kaliumsulfidlösung in derselben Weise einwirken lässt, wie es oben bei der Darstellung des naphtalinthiosulfonsauren Salzes beschrieben ist. Man erbält hier eine alkoholische Lösung, die nach dem Abfiltriren des Chlorkaliums schöne weisse Krystalle abscheidet. Leider ist auch hier die Schwierigkeit einer Reinigung vorhanden, da das gebildete Kaliumsalz sich nur aus seiner concentrirten alkoholischen Lösung abscheidet und dann leicht die ganze Lösung zu einem Krystallbrei erstarrt. Dieser Krystallbrei wurde abgesaugt und durch Waschen mit Aether von der dunkel gefärbten Mutterlauge befreit. In Wasser ist das Salz leicht löslich.

Die Analyse des auf diese Weise gereinigten Salzes ergab, wie leicht erklärlich, einen zu hohen Kaliumgehalt, da eine vollständige Trennung des thiosulfonsauren Salzes von dem gebildeten Chlorkalium in der oben beschriebenen Weise unmöglich ist. 
Müller: Einwirk. v. Oxalessigester auf Guanidin etc. 475

0,6059 Grm. Substanz gaben 0,2448 Grm. $\mathrm{K}_{2} \mathrm{SO}_{4}$, entsprechend 0,1097 Grm. $\mathbf{K}=18,1 \% \mathrm{~K}$.

Die Formel $\mathrm{C}_{7} \mathrm{H}_{7} \mathrm{SO}_{2} \mathrm{SK}$ verlangt $17,3 \% \mathrm{~K}$.

Dass in den beschriebenen Salzen aber wirklich thiosulfonsaure Salze vorliegen, konnte einerseits durch Reactionen erkannt werden, wurde andrerseits aber auch durch eine Reihe von Synthesen, bei denen diese thiosulfonsauren Salze als Ausgangsmaterialien dienten, dargethan.

\section{Untersuchungen ans dem organischen Laboratorium der technischen Hochschule zu Dresden.}

\section{Einwirkung von 0xalessigester auf Guanidin und Harnstoffabkömmlinge ; \\ von \\ R. Müller.}

Einleitung. - Durch die ausführlichen Untersuchungen von Behrend und seinen Schülern gelang es, durch Condensation von Acetessigester und Harnstoff, sowie Thioharnstoff und Guanidin, Derivate des hypothetischen Uracils darzustellen.

R. Behrend ${ }^{1}$ ) erhielt bei der Einwirkung von Harnstoff auf Acetessigester zuerst ein Condensationsprodukt unter Austritt von Wasser:

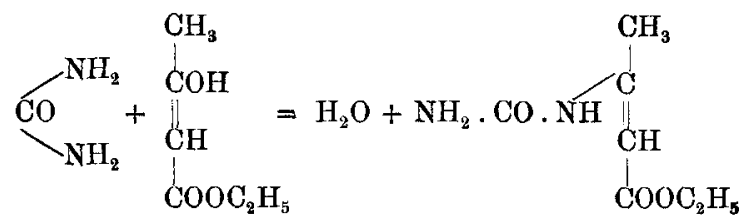

Das durch Verseifung erhaltene Natriumsalz wird durch Säuren nun condensirt, unter Austritt eines weiteren Moleküls Wasser und gleichzeitiger Ringschliessung:<smiles></smiles>

1) Ann. Chem. 229, 1. 